\title{
ON EXTREMAL POINTS OF THE UNIT BALL IN THE BANACH SPACE OF LIPSCHITZ CONTINUOUS FUNCTIONS
}

\author{
S. ROLEWICZ \\ (Received 1 June 1984; revised 12 February 1985) \\ Communicated by $\mathrm{H}$. Rubinstein
}

\begin{abstract}
It is shown that for arbitrary $\varepsilon>0$ there is a function $x(t, x)$ defined on the square $[0,1] \times[0,1]$ such that $x(t, s)$ represents an extremal point of the unit ball in the space of Lipschitz continuous functions, and the gradient of $x(t, s)$ is equal to 0 except on a set of measure at most $\varepsilon$.
\end{abstract}

1980 Mathematics subject classification (Amer. Math. Soc.): 46 A 55, 52 A 07.

Keywords and phrases: convexity, Choquet theory, convex sets in topological vector spaces.

The investigations of Lipschitz observability and theorems concerning forms of optimal observations [1] stimulate investigations of extremal points of the unit ball in the space of Lipschitz functions defined on a metric space $\Omega$ with a metric $\rho$, and with the classical Lipschitz distance

$$
d(f, g)=\sup _{t, t_{1} \in \Omega} \frac{\left|[f(t)-g(t)]-\left[f\left(t_{1}\right)-g\left(t_{1}\right)\right]\right|}{\rho\left(t, t_{1}\right)} .
$$

The semimetric $d$ is invariant, and hence it induces a seminorm

$$
\|f\|=d(f, 0)=\sup _{t, t_{1} \in \Omega} \frac{\left|f(t)-f\left(t_{1}\right)\right|}{\rho\left(t, t_{1}\right)} .
$$

Observe that the semimetric $d$ and the seminorm \|\| do not distinguish the functions which differ by a constant. In fact, we consider the quotient space $L / C$

(c) 1986 Australian Mathematical Society $0263-6115 / 86 \$ A 2.00+0.00$ 
of all Lipschitz continuous functions $L$ divided by the space of constant functions $C$. The seminorm \|\| induces a norm on $L / C$, and $L / C$ is a Banach space with this norm.

In further considerations it will be easier for us to consider an isomorphic and isometric image of $L / C$, namely the space $L_{a}$, where $a \in \Omega$, of all Lipschitz functions vanishing at $a$. Equation (2) above defines the isometric norm on $L_{a}$.

If $\Omega$ is an interval [ $\alpha, \beta]$, then each function $f$ belonging to $L_{a}, \alpha \leqslant a \leqslant \beta$, is differentiable almost everywhere. If $f$ is an extremal point of the unit ball in $L_{a}$, then its derivative $f(t)$ has modulus equal to one almost everywhere; $|f(t)|=1$ a.e. [1].

It is interesting that a similar theorem does not hold in the case when $\Omega$ is the square $[0,1] \times[0,1]$. Of course in this case also, every Lipschitz function is differentiable almost everywhere. However we have

THEOREM 1. For each $\varepsilon>0$, there is a function $f \in L_{a}$, being an extremal point of the unit ball of $L_{a}$, such that the support of the gradient has Lebesgue measure less than $\mathrm{\varepsilon}$

$$
\left|\left\{t:\left.\nabla f\right|_{t} \neq 0\right\}\right|<\varepsilon,
$$

where $|E|$ denotes the two dimensional Lebesgue measure of a set $E$.

The proof of Theorem 1 is based on the following notions and lemmas. Let $\Omega$ be a connected metric space. Let $\left\{\Omega_{\alpha}\right\}$ be a covering of $\Omega$, i.e. $\cup_{\alpha} \Omega_{\alpha} \supset \Omega$. We say that the covering $\left\{\Omega_{\alpha}\right\}$ is finitely connected if each of the sets $\Omega_{\alpha}$ is connected, and if, for arbitrary $t, \bar{t} \in \Omega$, there is a finite system of sets $\Omega_{\alpha_{1}}, \ldots, \Omega_{\alpha_{n}}$ for which there are $t_{0}=t, t_{1}, t_{2}, \ldots, t_{n}=\bar{t}$ such that $t_{i-1}, t_{i} \in \Omega_{\alpha_{i}}$.

LEMMA 1. Let $\left\{\Omega_{\alpha}\right\}$ be a finitely connected covering of $\Omega$. Let $f$ be an arbitrary Lipschitz function of seminorm 1 defined on $\Omega$. Suppose that for all $\alpha$ the restriction f to $\Omega_{\alpha}, f \mid \Omega_{\alpha}$, is an extremal point of the unit ball of the space of Lipschitz functions defined on $\Omega_{\alpha}$. Then $f$ is an extremal point of the space of Lipschitz functions defined on $\Omega$.

Proof. Suppose that $f$ is not an extremal point of the unit ball of the space of Lipschitz functions defined on $\Omega$. Then by definition there are two Lipschitz functions $f_{1}, f_{2}$ with $\left\|f_{1}\right\|=\left\|f_{2}\right\|=1$ such that $f_{1} \neq f_{2}$ and $\left(f_{1}+f_{2}\right) / 2=f$. This leads to a contradiction. Take any two points $t$ and $t$ such that

$$
f_{1}(t)-f_{2}(t) \neq f_{1}(\bar{t})-f_{2}(\bar{t}) \text {. }
$$

Such points must exist since $f_{1} \neq f_{2}$. Since the covering $\Omega_{\alpha}$ is finitely connected, there is a system of points $t_{0}=t, t_{1}, \ldots, t_{n}=\bar{t}$ and a system of indices $\alpha_{1}, \ldots, \alpha_{n}$ 
such that $t_{i-1}, t_{i} \in \Omega_{\alpha_{i}}, i=1,2, \ldots, n$. Since the restrictions of $f$ to the $\Omega_{\alpha_{i}}$ are extremal points of the unit ball in the space of Lipschitz functions, and since $f_{1} \mid \Omega_{\alpha_{i}}$ and $f_{2} \mid \Omega_{\alpha_{i}}$ have for each $\alpha_{i}$ a pseudonorm not greater than one, the function $f_{1}(t)-f_{2}(t)$ is constant on each $\Omega_{\alpha_{i}}$. This implies that

$$
f_{1}\left(t_{0}\right)-f_{2}\left(t_{0}\right)=f_{1}\left(t_{1}\right)-f_{2}\left(t_{1}\right)=\cdots=f_{1}\left(t_{n}\right)-f_{2}\left(t_{n}\right),
$$

and this contradicts (4).

Proof of Theorem 1. Take an arbitrary $\delta>0$. Now take the Cantor set $K_{\delta} \subset[0,1]$ of measure $1-\delta$. This set we obtain by the classical Cantor construction changed in such a way that, at the $n$th step, we remove from each interval the central interval of length $(\delta /(1+\delta))^{n}$. The complement of $K_{\delta}, G^{\delta}=[0,1] \backslash K_{\delta}$, is an open set of measure equal to $\delta$, and it is dense in $[0,1]$. Let

$$
\Omega=\left(G^{\delta} \times[0,1]\right) \cup\left([0,1] \times\left[\frac{1}{2}-\delta, \frac{1}{2}+\delta\right]\right) .
$$

It is easy to see that the set $\Omega$ is connected and dense in the whole square $[0,1] \times[0,1]$. Now we shall define a finitely connected covering in the following way. We enumerate all the components of $G^{\delta}$. They are intervals $\left(a_{n}, b_{n}\right)$. Now we define sets

$$
\begin{aligned}
& \Omega_{1, n}=\left\{(x, y): a_{n}<x<b_{n}, 0 \leqslant y \leqslant \frac{1}{2}-\delta+\frac{1}{2}\left(b_{n}-a_{n}\right)-\left|x-\frac{a_{n}+b_{n}}{2}\right|\right\}, \\
& \Omega_{2, n}=\left\{(x, y): a_{n}<x<b_{n}, 1 \geqslant y \geqslant \frac{1}{2}+\delta-\frac{1}{2}\left(b_{n}-a_{n}\right)+\left|x-\frac{a_{n}+b_{n}}{2}\right|\right\}
\end{aligned}
$$

and

$$
\Omega_{0}=\operatorname{cl}\left([0,1] \times\left[\frac{1}{2}-\delta, \frac{1}{2}+\delta\right] \backslash \bigcup_{\substack{n=1 \\ j=1,2}} \Omega_{j, n}\right) .
$$

Since $\Omega_{0}$ is closed, the sets

$$
\Omega_{0}, \Omega_{1, n}, \Omega_{2, n}, \Omega_{1,2}, \Omega_{2,2}, \ldots
$$

constitute a finitely connected covering. Now we define a function $f(x, y)$ on $\Omega$ in the following way:

$$
f(x, y)=\left\{\begin{array}{l}
\frac{a_{n}+b_{n}}{2}-\left|\frac{a_{n}+b_{n}-2 x}{2}\right| \text { for }(x, y) \in \Omega_{j, n}, j=1,2, \\
\delta-\left|y-\frac{1}{2}\right| \text { for }(x, y) \in \Omega_{0} .
\end{array}\right.
$$

It is easy to verify that $f(x, y)$ is a continuous function on $\Omega$ and that its restriction to $\Omega_{0}$ and $\Omega_{j, n}$ is, for each of these sets, an extremal point of the unit ball of the corresponding space of Lipschitz functions. Thus by Lemma 1 it is an 
extremal point of the unit ball of the Lipschitz functions defined on $\Omega$. Observe that if $(x, y) \in[0,1] \times[0,1]$, and if $(x, y)$ does not belong to $\Omega$, then for any sequence $\left(x_{n}, y_{n}\right) \in \Omega$ such that $\left(x_{n}, y_{n}\right)$ tends to $(x, y)$, we have $\lim f\left(x_{n}, y_{n}\right)=0$. Thus we can extend the function $f$ from $\Omega$ to a continuous function on the square $[0,1] \times[0,1]$ by taking

$$
\tilde{f}(x, y)= \begin{cases}f(x, y) & \text { for }(x, y) \in \Omega, \\ 0 & \text { for }(x, y) \notin \Omega .\end{cases}
$$

Since $\Omega$ is dense in $[0,1] \times[0,1]$, the extension is unique, and thus $\tilde{f}(x, y)$ is an extremal point of the unit ball of the Lipschitz functions defined on $[0,1] \times[0,1]$.

Observe that the gradient of $\tilde{f}(x, y)$ vanishes outside $\Omega$, and the set $\Omega$ has two dimensional Lebesgue measure less than $3 \delta$. Taking $\delta \leqslant \varepsilon / 3$, we obtain the theorem.

\section{References}

[1] S. Rolewicz, 'On optimal observability of Lipschitz systems', in Selected topics in operations research and mathematical economics, Lecture Notes in Economics and Mathematical Systems 226, Springer-Verlag, pp. 151-158.

Instytut Matematyczny

Polskiej Akademii Nauk, Sniadeckich 8

00950 Warszawa

Poland 